\title{
Interferência do microbioma gastrointestinal no ganho de peso e obesidade: uma revisão da literatura
}

Gastrointestinal microbiome interference without weight gain and obesity: a literature review

Interferencia del microbioma gastrointestinal sin aumento de peso y obesidad: revisión de la literatura

Eduarda Lemos de Oliveira Silva ${ }^{1 *}$, Nestor Augusto Avelino Leite ${ }^{2}$, Joao Victor Filgueiras Mota ${ }^{3}$, Igor Barcelos Thiengo ${ }^{4}$, Marcos de Oliveira Carneiro ${ }^{5}$, Janaína Pedroni Arpini ${ }^{1}$, Donato Moraes Dorna Foletto ${ }^{6}$, Letícia de Moraes Souza7, Gabriel Filipe dos Santos Oliveira ${ }^{8}$, Janyara Anny Azevedo de Andrade 9 .

\section{RESUMO}

Objetivo: Revisar as principais relações associadas entre o impacto da microbiota intestinal no ganho de peso e obesidade, bem como os fatores predisponentes dessa condição e seus respectivos tratamentos, visando a perda de peso e suas consequências benéficas ao alterar as colônias prevalentes e equilibrar a relação entre hospedeiro e comunidade microbiana. Revisão bibliográfica: A microbiota intestinal é repleta de bactérias que coexistem em simbiose, sendo que alterações nesse sistema podem influenciar no ganho de peso e no desenvolvimento e agravamento de doenças como obesidade e diabetes. Foi constatado que a secreção de hormônios intestinais orexígenos e anorexígenos, é modulada pela microbiota através de metabólitos ativos, com subsequente ativação de receptores relacionados à manutenção de tolerância a glicose e sensibilidade insulínica, construindo assim boa evidência dos impactos do biofilme intestinal nos centros do apetite. Considerações finais: Mais estudos são necessários para avaliar a antibioticoterapia e a suplementação de prebióticos e probióticos como modificadores do ambiente intestinal, com intuito de identificar os fatores de risco e de proteção.

Palavras-chave: Microbioma gastrointestinal, Ganho de peso, Obesidade.

\footnotetext{
ABSTRACT

1 União Educacional do Vale do Aço (UNIVAÇO), Ipatinga - MG.

*E-mail: eduardalemosoliveira@hotmail.com

${ }^{2}$ Faculdade de Medicina de Campos (FMC), Campos dos Goytacazes - RJ.

${ }^{3}$ Universidade Federal do Pará (UFPA), Belém - PA.

${ }^{4}$ Universidade Federal de Viçosa (UFV), Viçosa - MG.

${ }^{5}$ Universidade Federal do Acre (UFAC), Rio Branco - AC.

${ }^{6}$ Faculdade Brasileira Multivix (MULTIVIX), Vitória - ES.

7 Universidade Vila Velha (UVV), Vila Velha - ES.

8 Universidade Federal de Ouro Preto (UFOP), Ouro Preto - MG.

${ }^{9}$ Centro Universitário UniFacisa (UNIFACISA), Campina Grande - PB.
}

Objective: Review the main relationships associated between the impact of intestinal microbiota on weight gain and obesity, as well as the predisposing factors of this condition and their respective treatments, aiming at weight loss and its beneficial consequences by changing the prevalent colonies and balancing the relationship between host and microbial community. Bibliographic review: The intestinal microbiota is full of 
bacteria that coexist in symbiosis, and changes in this system can influence weight gain and the development and worsening of diseases such as obesity and diabetes. It was found that the secretion of orexigenic and anorectic intestinal hormones is modulated by the microbiota through active metabolites, with subsequent activation of receptors related to the maintenance of glucose tolerance and insulin sensitivity, thus building good evidence of the impacts of the intestinal biofilm on appetite centers. Final considerations: More studies are needed to evaluate antibiotic therapy and supplementation of prebiotics and probiotics as modifiers of the intestinal environment, in order to identify risk and protective factors.

Keywords: Gastrointestinal microbiome, Weight gain, Obesity.

\section{RESUMEN}

Objetivo: Revisar las principales relaciones asociadas entre el impacto de la microbiota intestinal en el aumento de peso y la obesidad, así como los factores predisponentes de esta afección y sus respectivos tratamientos, con el objetivo de la pérdida de peso y sus consecuencias beneficiosas al cambiar las colonias prevalentes y equilibrar la relación entre el huésped y la comunidad microbiana. Revisión bibliográfica: La microbiota intestinal está llena de bacterias que coexisten en simbiosis, y los cambios en este sistema pueden influir en el aumento de peso y el desarrollo y empeoramiento de enfermedades como la obesidad y la diabetes. Se encontró que la secreción de hormonas intestinales orexigénicas y anoréxicas es modulada por la microbiota mediante metabolitos activos, con la posterior activación de receptores relacionados con el mantenimiento de la tolerancia a la glucosa y la sensibilidad a la insulina, construyendo así una buena evidencia de los impactos del biofilm intestinal sobre el centro del apetito. Consideraciones finales: Se necesitan más estudios para evaluar la terapia antibiótica y la suplementación de prebióticos y probióticos como modificadores del ambiente intestinal, con el fin de identificar factores de riesgo y protectores.

Palabras clave: Microbioma gastrointestinal, Aumento de peso, Obesidad.

\section{INTRODUÇÃO}

Atualmente sabe-se que as doenças crônicas são as maiores causas de morbimortalidade no mundo, sendo a obesidade a mais incidente entre elas. Dados do Instituto Brasileiro de Geografia e Estatística (IBGE) mostram o aumento significativo da porcentagem de brasileiros acometidos pela obesidade e segundo 0 órgão, em 2020 houve um aumento de $14,6 \%$ dos casos em maiores de vinte anos, passando de 12,2\% para $26,8 \%$. Tais números demonstram um problema de saúde pública emergente e apesar de ser uma doença prevenível e tratável, a obesidade ainda onera o sistema devido às suas inúmeras complicações e prejuízos à qualidade de vida dos indivíduos (ABENAVOLI L, et al., 2019).

A obesidade é uma doença multifatorial que sofre influência de determinantes ambientais e genéticos, podendo estar associada a outras comorbidades, como diabetes mellitus tipo 2 , doenças cardiovasculares e hepáticas, câncer e síndrome metabólica. Recentemente descobriu-se que a microbiota intestinal está diretamente relacionada ao ganho de peso e à regulação do sistema imunológico e nervoso, influenciando o centro da fome e da saciedade localizados em diferentes sítios do hipotálamo (LEE CJ, et al., 2019)

Ademais, o intestino humano é repleto de bactérias que coexistem em simbiose, sendo os filos Firmicutes (60 a $80 \%$ ) e Bacterioides (10 a 30\%) os mais prevalentes. A atuação desse sistema em indivíduos susceptíveis associados ao desbalanço energético, são capazes de gerar o ganho de peso. Em contrapartida, a microbiota de pessoas consideradas eutróficas de acordo com o Índice de Massa Corpórea (IMC), contribui para a manutenção do equilíbrio da constituição física desses organismos. Essa discrepância entre as diferentes colônias de microorganismos advém de causas que se originam em diferentes momentos da vida, como na gestação com a alimentação materna, tipo de via de parto e uso indiscriminado de antibiótico nos primeiros anos de vida (ABENAVOLI L, et al., 2019; ALLAM-NDOUL B, et al., 2020).

Por conseguinte, novos tratamentos estão surgindo na tentativa de minimizar problemas futuros relacionados ao sobrepeso e obesidade. A suplementação com pré e probióticos e transplante fecal, 
representam o advento do tratamento diretamente relacionado ao controle microbiológico intestinal. Condutas comumentes usadas para controles de peso, como a cirurgia bariátrica, também contribuem para a mudança do padrão da flora intestinal de maneira indireta, corroborando para o reequilíbrio dessa interação (TORRESFUENTES C, et al., 2017).

Diante do exposto, o estudo apresenta como objetivo descrever as principais relações associadas entre o impacto da microbiota intestinal no ganho de peso e obesidade, bem como os fatores predisponentes dessa condição e seus respectivos tratamentos, visando a perda de peso e suas consequências benéficas ao alterar as colônias prevalentes e equilibrar a relação entre hospedeiro e comunidade microbiana.

\section{REVISÃO BIBLIOGRÁFICA}

\section{Definição microbiota}

A microbiota intestinal é constituída por colônias com cerca de 100 trilhões de microrganismos que vivem em condições de simbiose com seus hospedeiros humanos, sendo os filos Bacterioides e Firmicutes os mais predominantes. Essas bactérias desempenham papel importante na saúde da digestão, síntese de vitaminas, absorção de íons, regulação do apetite, metabolismo de ácidos graxos e glicose e agem como barreira imunológica (GÓROWSKA-KOWOLIK K e CHOBOT A, 2019). Rosenbaum M, et al. (2015) descreve a microbiota como órgão enteroendócrino responsivo, devido suas capacidades de secretar ou alterar a produção de moléculas que interferem no balanço energético e nos estoques de energia.

A composição das colônias de microrganismos na microbiota sofre modificações desde o início da vida, sendo influenciada por fatores como aumento da exposição a nutrientes, idade, tipo de dieta, uso de medicamentos, doenças inflamatórias e estado metabólico. Rosenbaum M, et al. (2015) constatou que existe uma variabilidade transversal na microbiota entre indivíduos, esta variabilidade é explicada pela influência destes fatores sobre as colônias. Dessa forma, conclui-se que a microbiota é uma parte inerente de cada indivíduo, podendo ser modificada e sofrer adaptações de acordo com as necessidades individuais.

O desequilíbrio na composição da microbiota humana ou disbiose foi relacionado com surgimento, manutenção e complicações de diversas doenças metabólicas, incluindo a obesidade. A proporção fracionária reduzida de espécies de Bacterioides em relação a Firmicutes é indicada como característica diferencial do microbioma de indivíduos obesos quando comparado a adultos eutróficos de acordo com o Índice de Massa Corpórea (IMC). Por conseguinte, estudos comprovaram que a diversidade microbiana é diminuída durante a obesidade e é aumentada quando se perde peso, sendo ponto importante para busca de tratamentos relacionados com esse achado (GÓROWSKA-KOWOLIK K e CHOBOT A, 2019).

\section{Relação entre microbiota intestinal e sistema nervoso central (Eixo Cérebro-Intestino)}

Diversos achados nas últimas décadas, tanto em modelos animais quanto em humanos, apontam forte relação entre Sistema Nervoso, Trato Gastrointestinal (TGI) e microbiota intestinal. Esse sistema complexo de interações pode ser chamado de eixo Cérebro-Intestino, e desempenha diversas funções neuroendócrinas e neuroimunes no metabolismo e homeostase corporal, servindo como uma via de comunicação dupla, capaz de autorregulação. É fator contribuinte e potencialmente causal no desenvolvimento de diversas patologias, como obesidade e diabetes (ARNETH BM, 2018).

Sua interdependência se relaciona a diversos mecanismos diferentes. Na revisão de literatura realizada por Carabotti M, et al. (2015), importantes formas de interação foram observadas. A produção e expressão de neurotransmissores (Ex.: Ácido gama-aminobutírico (GABA), serotonina, melatonina, acetilcolina, etc.), regulação da barreira epitelial intestinal através da integridade da zona de oclusão, secreção de metabólitos bacterianos bioativos, modulação de fibras entéricas sensoriais aferentes e impactos na resposta imune da mucosa, são alguns dos mecanismos que o intestino utiliza para se comunicar com o Sistema Nervoso Central (SNC). Por outro lado, estímulos centrais podem causar alterações na motilidade, permeabilidade intestinal, função imune, produção de muco e consequentemente alterações na composição da flora. 
É fato que o Nervo Vago (NV) apresenta papel central nessa comunicação, estabelecendo relação direta entre o Sistema Nervo Entérico (SNE) e o SNC. Na revisão de Forsythe P, et al. (2014) observou-se diversas formas de interação, porém com resultados neurológicos variados. Em alguns estudos a ativação vagal promoveu sintomas depressivos, já em outros atenuou tais sintomas. Em alguns outros estudos foram observados efeitos anxiogênicos e ansiolíticos. Fica claro, portanto, que a ação vagal não se limita estritamente a sua ativação/supressão, mas compõe um sistema complexo e não completamente compreendido.

Torres-Fuentes C, et al. (2017), em sua revisão, constatou que alguns estudos antigos em modelos animais, a vagotomia gerou redução no estímulo anorexígeno, levando ao aumento da ingestão alimentar e ganho de peso. Porém alguns outros trabalhos envolvendo estimulação vagal, apesar de apontarem para a tendência geral de mediação de sinais de saciedade pelo NV, principalmente em animais, não conseguiram reproduzir tais resultados de forma consistente quando realizados em humanos, evidenciando maior necessidade de esclarecimentos do papel vagal na ingestão calórica e ganho de peso (BODENLOS JS, et al., 2014).

Outra forma de atuação da microbiota, em conjunto com o SNC, no desenvolvimento e/ou agravamento da obesidade, é através de sinais bioquímicos provenientes do metabolismo microbiano e sua interação com os centros de fome/saciedade cerebrais. Zanchi D, et al. (2017), caracteriza duas principais famílias de hormônios gastrointestinais, os orexigênicos e os anorexígenos. Da primeira faz parte a grelina, e da segunda hormônios como, Peptídeo semelhante a glucagon 1 (GLP-1), peptídeo tirosina tirosina (PYY), colecistocinina (CCK) e leptina. Após revisão de estudos de neuroimagem aliado à análise hormonal plasmática, foi constatado associação direta entre concentração sanguínea dessas classes de hormônios com modificações em regiões cerebrais associadas ao circuito neural do apetite.

Foi constatado que a secreção de tais hormônios é modulada pela microbiota através de metabólitos ativos, em especial Ácidos Graxos de Cadeia Curta (AGCC). Também é implicado o uso de ácidos biliares e seus conjugados pelos microorganismos, com subsequente ativação de receptores como o Farnesoid $X$ Receptor (FXR) e o Takeda G-Protein-Coupled Receptor 5 (TGR5), relacionados a manutenção de tolerância a glicose e sensibilidade insulínica no intestino e fígado. Esses mecanismos moduladores são responsáveis por regular a secreção gastrointestinal desses hormônios pelo SNE na corrente sanguínea. E quando associados ao papel vagal na comunicação cérebro-intestino, constroem boa evidência dos impactos do biofilme intestinal nos centros do apetite (TORRES-FUENTES C, et al., 2017).

Ademais, outro importante mecanismo neuroendócrino de interação é através da ação de neurotransmissores, como o Ácido Gama-Aminobutírico (GABA), serotonina (5-HT), melatonina, acetilcolina e histamina, moduladas diretamente através da ação da flora. Em estudos com camundongos livres de germes foram observados níveis séricos diminuídos de GABA e serotonina. A 5-HT em especial, é produzida em sua grande maioria no TGI ( 90\%), e apesar de ainda não completamente estabelecido seu processo de metabolismo e funcionamento, está clara a influência dos microorganismos. As demais funções da $5-\mathrm{HT}$, como o controle da motilidade intestinal, além de fatores relacionados a sua produção e manutenção, tanto no SNC quanto no TGI são intrinsecamente associadas ao eixo cérebro-microbiota-intestinal (SAMPSON TR e MAZMANIAN SK, 2015).

\section{Microbiota, sobrepeso e obesidade}

Algumas pesquisas puderam comprovar a influência das alterações na microbiota intestinal no desenvolvimento de obesidade e diabetes tipo 2 (DT2). Entretanto, tal mecanismo de interferência e correlação ainda é pouco sabido. Na pesquisa de Scheithauer T, et al. (2020), os autores concluíram de que há fortes evidências de que a interação tripartida entre a microbiota intestinal, o sistema imunológico do hospedeiro e o metabolismo é um participante crítico na fisiopatologia da obesidade e DT2. Outrossim, levando em consideração tal informação, algumas colônias de bactérias entéricas devem ser analisadas, no que tange a sua interferência ou não no desenvolvimento da obesidade e DT2 (ABENAVOLI L, et al., 2019; ALLAM-NDOUL B, et al., 2020). 
Muitas bactérias compõem o trato intestinal de um organismo vivo. No ser humano, é observada a presença de Clostridium difficile, Escherichia coli, Lactobacillus rhamnosus GG e demais espécies. Ademais, em alguns estudos recentes (MAGNE F, et al., 2020), a obesidade e DT2 estão sendo associadas a uma abundância relativa mais baixa de bactérias do gênero Bacteroides e Bifidobacterium, como também produtoras de butirato Faecalibacterium prausnitzii (SALGUERO MV, et al., 2019). Sendo assim, torna-se evidente que a contribuição da microbiota para o ganho e perda de peso é inegável e muito mais complexa do que um simples desequilíbrio (ALLAM-NDOUL B, et al., 2020).

Além disso, a microbiota intestinal atua como imunomodulador, ou seja, tanto na fase de desenvolvimento da DT2 como na influência à obesidade, essa microbiota é um fator impulsionador para a metainflamação, como também de problemas metabólicos no que tange à obesidade (SALGUERO MV, et al., 2019). A inflamação é bastante observada em pacientes obesos, apresentando maior tônus inflamatório e asma grave, em que essa inflamação influencia na sensibilização de insulinas e na função de células betas (MAGNE F, et al., 2020).

Dessa forma, algumas pesquisas desenvolveram formas alternativas de intervenções farmacológicas para a redução dessa inflamação, atuando principalmente na tolerância da glicose (LU J, et al., 2019). Sendo assim, estas incluem a nova citocina híbrida interleucina (IL), que é produzida em uma Escherichia coli geneticamente modificada pela fusão de IL-2 murina e IL-33 murina (SABAPATHY V, et al., 2019). Outras estratégias de tratamento incluem o bloqueio do receptor de IL-1, antagonismo de IL-1 $\beta$, inibição da via NFKB pró-inflamatória intracelular e antagonismo de TNF (CAVELTI-WEDER C, et al., 2012).

Na pesquisa de Hong EG, et al. (2009), os autores observaram que a superexpressão de IL-10 no tecido muscular murino melhorou a sensibilidade à insulina, mesmo em condições de dieta rica em gordura. Além disso, a carga viral no intestino também pode influenciar nessas alterações metabólicas. Norovírus e rotavírus são altamente prevalentes no trato gastrointestinal (ESTES MK, et al., 2008)

\section{Microbiota usada na prevenção e tratamento da obesidade}

Os probióticos são microrganismos vivos com a capacidade de alterar a fisiologia do sistema gastrointestinal aumentando a diversidade de microrganismos e modulando o sistema imunológico. Eles podem desempenhar papel importante na homeostase corporal inibindo o crescimento de microrganismos patológicos, ajudando na prevenção e tratamento de doenças do trato gastrointestinal (WAGNER NRF, et al., 2018).

Os termos microrganismos e probióticos se confundem. Para o melhor entendimento para que um microrganismo seja definido como probiótico ele deve atender critérios como: não ser patogênico, possuir a capacidade de persistir no trato gastrointestinal, aderir ao epitélio, ser originário da flora humana, e ter a capacidade de influenciar na atividade do sistema digestivo (PEREIRA AAR, et al., 2019).

A ação dos probióticos no combate a obesidade envolve vários mecanismos, mas pode se evidenciar basicamente três: Imunomodulação, atividade antimicrobiana e função de barreira. Brusaferro A, et al. (2018) analisaram o efeito favorável para redução do peso corporal, diminuição da massa gorda, redução dos marcadores inflamatórios de camundongos através da administração de probióticos. Percebeu-se também que certos probióticos podem exercer efeito antagonista a perda de peso exacerbando o ganho de peso, exigindo maior controle, principalmente das espécies e cepas administradas.

Kadooka YO, et al. (2010), realizaram ensaio duplo-cego, multicêntrico, randomizado e controlado por placebo em adultos com Índice de Massa Corporal (IMC) e área de gordura visceral abdominal mais elevados, e administraram leite fermentado contendo Lactobacillus gasseri LG2055 ou apenas leite fermentado por 12 semanas. Os resultados mostraram que os pacientes expostos ao probiótico obteve redução de 4,6\% e 3,3\% da gordura abdominal e visceral, respectivamente. Observou-se também redução no IMC 1,5\% e no peso corporal de 1,4\%. Em contrapartida, não se notou nenhuma alteração no grupo controle.

Abenavoli L, et al. (2019) analisaram estudos relacionados à ação dos probióticos na faixa etária pediátrica, em adolescentes e adultos. Os dados do artigo evidenciaram que misturas de microrganismo selecionados tem poder efetivo em reduzir fatores ligados à obesidade em determinadas faixas etárias, reduzindo o IMC, gordura no fígado e diminuindo a resistência à insulina. 


\section{Prebióticos}

Segundo a Organização Mundial de Gastrenterologia, prebióticos são fórmulas alimentares que não sofrem digestão e afetam positivamente a microbiota intestinal do indivíduo estimulando e/ou selecionando o crescimento de microrganismo específicos que já colonizam o trato gastrointestinal. Eles são responsáveis pela modulação de funções fisiológicas importantes a exemplo da absorção de cálcio, composição da flora intestinal e possivelmente do metabolismo lipídico (SAAD SMI, 2006).

Wang $Y$, et al. (2016), realizaram estudo controle randomizado com 30 indivíduos entre homens e mulheres de 27 a 78 anos com (IMC) de $20-40 \mathrm{Kg} / \mathrm{m}^{2}$ e com hipercolesterolemia. O estudo evidenciou aumento na contagem de bacteroides da microbiota intestinal durante a suplementação com $3 \mathrm{~g} / \mathrm{d}$ de $\beta$ Glucano HMW em comparação com o grupo controle. Houve redução da circunferência da cintura, triglicerídeos (TG), proteínas de baixa densidade (LDL), níveis de glicose e pressão arterial.

Aoe SE, et al. (2017), evidenciaram em seu estudo randomizado, duplo cego com pacientes que possuíam sobrepeso ou eram obesos e tinham circunferência da cintura de $\geq 85 \mathrm{~cm}$ para homens e $\geq 90 \mathrm{~cm}$ para mulheres. Nesses pacientes foram administrados dois pacotes de alimentos compostos por arroz e aveia, 0 grupo de intervenção foi enriquecido com $2,2 \mathrm{~g} \beta$-Glucano. Notou- se diminuição significativa do IMC, peso corporal, circunferência da cintura e área de gordura visceral em ambos os grupos os de controle e de intervenção.

\section{Simbióticos}

É uma mistura de microrganismos selecionados (probióticos) e ingredientes alimentares com a capacidade de estimular determinados germes da flora intestinal (prebióticos). O efeito de cada um: (probióticos) e (prebióticos) pode ser potencializado quando empregados juntos, justificando o emprego da terapia para alcançar melhores resultados contra a obesidade (CERDÓ T, et al., 2019).

\section{Uso de antibióticos e alterações da microbiota intestinal}

O uso indiscriminado dos antibióticos traz relevantes alterações na microbiota intestinal, os efeitos adversos incluem a resistência aos antibióticos, a dominação intestinal por bactérias patogênicas, a perda transitória ou profunda da diversidade microbiana, a suscetibilidade aumentada e prolongada a infecções, entre outros (KIM S, et al., 2017).

A respeito dos antibióticos é válido apontar que seu uso indiscriminado apresenta íntima relação com as alterações na microbiota intestinal e obesidade, apesar de ainda não estar totalmente esclarecida. Os mecanismos pelos quais se acredita que os antimicrobianos podem suscitar o ganho de peso são o aumento da capacidade da extração de polissacarídeos indigestíveis pelas bactérias intestinais, a redução de bactérias que atuam contra a obesidade, as alterações na lipogênese hepática e a depleção na defesa intestinal e nas vias imuno-metabólicas benéficas. Por fim, manter a microbiota intestinal do hospedeiro em equilíbrio é essencial visto que as disbioses causadas por antimicrobianos resultam em distúrbios metabólicos, como a obesidade (VALLIANOU N, et al., 2021).

\section{CONSIDERAÇÕES FINAIS}

A partir dos estudos anteriores, é possível observar que a microbiota possui a capacidade de ser alterada, de forma a se adaptar diante das necessidades de cada indivíduo. Para mais, destaca-se que é possível inferir que mudanças na microbiota intestinal influenciam no desenvolvimento de obesidades e até diabetes tipo 2. Nesse sentido, é importante que haja mais estudos que busquem identificar quais mecanismo fisiopatológicos que podem levar a microbiota intestinal a interferir no surgimento de tais doenças. Além disso, é de grande importância novas pesquisas que avaliem a suplementação de prebióticos ou probióticos na modificação de determinadas espécies de micro-organismos no ambiente intestinal com intuito identificar fatores de risco ou de proteção para o desenvolvimento dessas doenças. 


\section{REFERÊNCIAS}

1. ABENAVOLI L, et al. Gut Microbiota and Obesity: A Role for Probiotics. Nutrients, 2019; 11(11): 1-27.

2. ALLAM-NDOUL B, et al. Gut Microbiota and Intestinal Trans-Epithelial Permeability. International journal of molecular sciences, 2020; 21(17): 6402 .

3. AOE S, et al. Effects of high $\beta$-glucan barley on visceral fat obesity in Japanese individuals: A randomized, doubleblind study. Nutrition, 2017; 42: 1-6.

4. ARNETH BM. Gut-brain axis biochemical signalling from the gastrointestinal tract to the central nervous system: gut dysbiosis and altered brain function. Postgraduate Medical Journal, 2018; 94(1114): 446-452.

5. BODENLOS JS, et al. Vagus nerve stimulation and food intake: effect of body mass index. Journal of diabetes science and technology, 2014; 8(3): 590-595.

6. BRUSAFERRO A, et al. Is It Time to Use Probiotics to Prevent or Treat Obesity? Nutrients, 2018; $10(11): 1613$.

7. CARABOTTI M, et al. The gut-brain axis: interactions between enteric microbiota, central and enteric nervous systems. Annals of Gastroenterology, 2015; 28(2): 203-209.

8. CAVELTI-WEDER C, et al. Effects of Gevokizumab on Glycemia and Inflammatory Markers in Type 2 Diabetes. Diabetes Care, 2012; 35(8): 1654-1662.

9. CERDÓ T, et al. The Role of Probiotics and Prebiotics in the Prevention and Treatment of Obesity. Nutrients, 2019; 11(3): 635.

10. ESTES MK, et al. Simpósios da Fundação Novartis. John Wiley \& Sons, 2008; 82-100.

11. FORSYTHE P, et al. Vagal Pathways for Microbiome-Brain-Gut Axis Communication. Advances in Experimental Medicine and Biology, 2014; 817: 115-133.

12. GÓROWSKA-KOWOLIK K, CHOBOT A. The role of gut microbiome in obesity and diabetes. World Journal of pediatrics, 2019; 15: 332-340.

13. HONG EG, et al. Interleukin-10 prevents diet-induced insulin resistance by attenuating macrophage and cytokine response in skeletal muscle. Diabetes, 2009; 58(11): 2525-2535.

14. KADOOKA Y, et al. Effect of Lactobacillus gasseri SBT2055 in fermented milk on abdominal adiposity in adults in a randomised controlled trial. British Journal of Nutrition, 2013; 110: 1696-703

15. KIM S, et al. The intestinal microbiota: Antibiotics, colonization resistance, and enteric pathogens. Immunological Reviews, 2017; 279(1): 90-105.

16. LEE CJ, et al. Gut microbiome and its role in obesity and insulin resistance. Annals of the New York Academy of Science, 2020; 1461(1): 37-52.

17. LU J, et al. Adipose Tissue-Resident Immune Cells in Obesity and Type 2 Diabetes. Frontiers in immunology, 2019; 10: 1173.

18. MAGNE F, et al. The Firmicutes/Bacteroidetes Ratio: A Relevant Marker of Gut Dysbiosis in Obese Patients? Nutrients, 2020; 12(5): 1474.

19. PEREIRA AAR, et al. Probióticos e prebióticos na prevenção e tratamentos de doenças. Revista Brasileira Multidisciplinar, 2019; 22(3): 162-176.

20. ROSENBAUM M, et al. The gut microbiota in human energy homeostasis and obesity. Trends in Endocrinology \& Metabolism, 2015; 26(9): 493-501.

21. SAAD, SMI. Probióticos e Prebióticos: o Estado da Arte. Revista Brasileira de Ciências Farmacêuticas, 2006; 42(1): $1-16$.

22. SABAPATHY V, et al. Novel Immunomodulatory Cytokine Regulates Inflammation, Diabetes, and Obesity to Protect From Diabetic Nephropathy. Frontiers in Pharmacology, 2019;10: 572.

23. SALGUERO MV, et al. Dysbiosis of Gram-negative gut microbiota and the associated serum lipopolysaccharide exacerbates inflammation in type 2 diabetic patients with chronic kidney disease. Experimental and therapeutic medicine, 2019; 18(5): 3461-3469.

24. SAMPSON TR, MAZMANIAN SK. Control of brain development, function, and behavior by the microbiome. Cell Host \& Microbe, 2015; 17(5): 565-576.

25. SCHEITHAUER TPM, et al. Gut Microbiota as a Trigger for Metabolic Inflammation in Obesity and Type 2 Diabetes. Frontiers in immunology, 2020; 11: 571731.

26. TORRES-FUENTES C, et al. The microbiota-gut-brain axis in obesity. The Lancet Gastroenterology \& Hepatology, $2017 ; 2(10): 747-756$.

27. VALLIANOU N, et al. Do Antibiotics Cause Obesity Through Long-term Alterations in the Gut Microbiome? A review of Current Evidence. Current Obesity Reports, 2021; 1-19.

28. WAGNER NRF, et al. Mudanças na microbiota intestinal e uso de probióticos no pós-operatório de bypass gástrico em Y-de-Roux e gastrectomia vertical sleeve: uma revisão integrativa. ABCD Arquivos Brasileiros de Cirurgia Digestiva, 2018; 31(4): 1400.

29. WANG Y, et al. High molecular weight barley $\beta$-glucan alters gut microbiota toward reduced cardiovascular disease risk. Front Microbiol, 2016; 7: 129.

30. ZANCHI D, et al. The impact of gut hormones on the neural circuit of appetite and satiety: A systematic review. Neuroscience and biobehavioral reviews, 2017; 80: 457-475. 